\section{CITAÇÃO}

Tavares, JN (2017)

Modelo SIR em epidemiologia,

Rev. Ciência Elem., V5(02):020.

doi.org/10.24927/rce2017.020

\section{EDITOR}

José Ferreira Gomes,

Universidade do Porto

\section{RECEBIDO EM}

28 de maio de 2017

\section{ACEITE EM}

07 de junho de 2017

\section{PUBLICADO EM}

31 de junho de 2017

\section{COPYRIGHT}

(C) Casa das Ciências 2017.

Este artigo é de acesso livre, distribuído sob licença Creative

Commons com a designação CC-BY-NC-SA 4.0, que permite a utilização e a partilha para fins não comerciais, desde que citado 0 autor e a fonte original do artigo.

rce.casadasciencias.org

\title{
Modelo SIR em epidemiologia
}

\author{
João Nuno Tavares \\ CMUP/ Universidade do Porto \\ jntavar@fc.up.pt
}

Neste pequeno texto, procura-se ilustrar, através de um modelo simples (SIR), como a Matemática pode serútil na previsão da evolução de uma epidemia e na tomada de decisão sobre estratégias de combate à sua propagação (vacinação, quarentenas, etc.).

\section{INTRODUÇÃO}

1.1. Ao longo dos séculos, tem havido muitos exemplos de epidemias de várias doenças com efeitos dramáticos na população humana (e não só). Uma das mais conhecidas é a Peste Negra na Europa no século XIV ${ }^{1}$, que dizimou entre 25 a 75 milhões de pessoas (cerca de um terço da populacão europeia da altura!). Nos tempos coloniais, a propagação de doenças europeias, tais como o sarampo e a varíola, teve um impacto desastroso sobre certas populacões indígenas que não tinham desenvolvido resistência a essas doenças.

Hoje em dia, existem ainda exemplos trágicos - a SIDA, o vírus Ébola, o ZIKA e muitos outros.

\section{Se conseguirmos compreender como uma doença se propaga numa determinada} população, então estaremos melhor equipados para a conter, através de vacinação ou quarentena.

1.2. Muitas doenças são propagadas por indivíduos infectados que, por contacto com in- divíduos susceptíveis, os contagiam. Estas incluem gripe, sarampo, varicela, febre glandular e SIDA. Por outro lado, a malária é transmitida por meio de um hospedeiro, um mosquito, que transporta a doença de indivíduo para indivíduo. Algumas doenças são mais contagiosas do que outras. Sarampo e gripe são altamente contagiosas, enquanto a febre glandular é muito menos. Muitas doenças, tais como papeira e sarampo, conferem uma imunidade ao longo da vida; no entanto, gripe e febre tifóide têm períodos curtos de imunidade e podem ser contraídas mais do que uma vez.

1.3. Comecemos por esclarecer alguns conceitos prévios que surgem recorrentemente na modelação de epidemias.

O período de incubação da doença é o tempo entre a infecção e a aparência visível de sintomas. Isto não deve ser confundido com o 
- período de latência, que é o período de tempo entre a infecção e a capacidade de infectar alguém por contágio.

O período de latência é mais curto do que o período de incubação - um indivíduo pode estar a propagar a doença, sem ter até consciência de que a tem! Para o sarampo, o período de incubação é de cerca de 2 semanas e o período de latência é aproximadamente 1 semana.

Vamos neste Módulo aprender a modelar matematicamente a dinâmica da propagação de uma doença (gripe, para sermos mais concretos), adoptando algumas hipóteses simplificadoras (como aliás se faz em qualquer modelo matemático) que serão enunciadas já de seguida.

\section{HIPÓTESES DE MODELAÇÃO}

2.1. Vamos modelar a dinâmica de uma doença infecciosa (gripe, por exemplo), que se propaga numa certa população (alunos de uma escola por exemplo).

Para isso, começamos por dividir a população em 3 grupos ou classes:

- $\mathbf{S}=$ [Susceptíveis $]$ - os que podem apanhar gripe, mas que actualmente não estão infectados;

- I = [Infecciosos $]$ - os que estão infectados com gripe e podem contagiá-la a outros;

- $\quad \mathbf{R}=$ [Removidos] - os que não podem apanhar gripe, ou porque recuperaram permanentemente e ficaram imunes (pelo menos durante o período em análise), ou porque são naturalmente imunes ou porque morreram!

2.2. Por ser mais simples, vamos modelar a dinâmica da doença, isto é, a evolução temporal dos tamanhos de cada uma das classes acima referidas, usando tempo discreto:

$$
t_{0}, t_{1}, t_{2}, t_{3}, \cdots, t_{\mathrm{n}}, t_{\mathrm{n}+1}, \cdots
$$

0 intervalo entre dois períodos consecutivos $\Delta t=t_{n+1}-t_{n^{\prime}} n=0,1,2,3, \cdots$ supõe-se constante - é medido numa unidade de tempo apropriada (hora, dia, semana, ano, etc., conforme o contexto).

Portanto

$$
t_{n+1}=t_{n}+\Delta t, n=0,1,2, \cdots
$$

2.3. Seja $t \in\left[t_{0}, t_{1}, t_{2}, t_{3}, \cdots\right]$ um qualquer dos instantes acima referidos, e representemos por $S(t), I(t)$ e $R(t)$ o número de indivíduos, no instante $t$, em cada um dos grupos $\mathbf{S}$, I e $\mathbf{R}$, respectivamente. A modelação da forma como evoluem estes grupos, em interacção uns com os outros, conduzirá a um sistema acoplado de equações.

Matematicamente é mais conveniente manter o número total de indivíduos constante, e por isso contamos todos os indivíduos mortos em R. Assim, supomos que

$$
S(t)+I(t)+R(t) \equiv N(\text { constante) }
$$

para todo o $t \in\left[t_{0}, t_{1}, t_{2}, t_{3}, \cdots\right]$. Em geral pomos $t_{0}=0$. 
2.4. As hipóteses do modelo são as seguintes:

H1 A população em análise é muito numerosa o que permite ignorar efeitos aleatórios, devidos às diferenças entre os indivíduos e outros factores.

H2 Também assumimos que, em qualquer momento, a população é homogeneamente misturada, isto é, supomos que os infecciosos (infectados e contagiosos) e susceptíveis estão sempre distribuídos aleatoriamente na área em que a população vive (não se consideram eventuais heterogeneidades espaciais).

H3 A doenca é transmitida por proximidade ou contacto entre um infeccioso e um susceptível.

H4 Um susceptível fica infectado logo após a transmissão, isto é, ignoramos o período de latência da doença, fixando-o igual a zero.

H5 Os infectados eventualmente recuperam e, uma vez recuperados, ficam para sempre imunes (pelo menos durante o periodo em análise).

H6 Ignoramos nascimentos, mortes ou efeitos migratórios.

2.5. Consideremos agora um intervalo de tempo $[t, t+\Delta t]$, onde $t \in\left[t_{0}, t_{1}, t_{2}, t_{3}, \cdots\right]$, e representemos por

$$
\begin{aligned}
& \Delta S(t)=S(t+\Delta t)-S(t)=\left\{\begin{array}{c}
\text { Variação do número de suscetíveis } \\
\text { no intervalo }[t, t+\Delta t]
\end{array}\right] \\
& \Delta I(t)=I(t+\Delta t)-I(t)=\left[\begin{array}{c}
\text { Variação do número de infetados } \\
\text { no intervalo }[t, t+\Delta t]
\end{array}\right] \\
& \Delta R(t)=R(t+\Delta t)-R(t)=\left\{\begin{array}{c}
\text { Variação do número de recuperados } \\
\text { no intervalo }[t, t+\Delta t]
\end{array}\right]
\end{aligned}
$$

É claro que

$$
\begin{aligned}
& \Delta S(t)=-\left\{\begin{array}{c}
\text { Número de suscetíveis } \\
\text { infetados no intervalo }[t, t+\Delta t]
\end{array}\right] \\
& \Delta I(t)=-\left\{\begin{array}{c}
\text { Número de susceptíveis } \\
\text { infetados no intervalo }[t, t+\Delta t]
\end{array}\right]-\left[\begin{array}{c}
\text { Número de infetados } \\
\text { recuperados no intervalo }[t, t+\Delta t]
\end{array}\right] \\
& \Delta R(t)=-\left\{\begin{array}{c}
\text { Número de infetados } \\
\text { recuperados no intervalo }[t, t+\Delta t]
\end{array}\right]
\end{aligned}
$$

2.6. Vamos calcular em primeiro lugar o $\left\{\begin{array}{c}\text { Número de infetados } \\ \text { recuperados no intervalo }[t, t+\Delta t]\end{array}\right]$. Este número não 
deve depender do número de indivíduos suscetíveis $S(t)$, mas apenas do número de infetados $I(t)$. No nosso modelo, assumimos que esse número (de infetados recuperados) é directamente proporcional ao número de infetados $I(t)$ e também à duração do intervalo de tempo $\Delta t$. Escrevemos pois

$$
\left\{\begin{array}{c}
\text { Número de infetados } \\
\text { recuperados no intervalo }[t, t+\Delta t]
\end{array}\right\}=\gamma I(t) \Delta t
$$

onde $\gamma$ é uma constante positiva de proporcionalidade, chamada taxa de recuperação ou taxa de remoção. $\gamma$ é uma taxa per-capita e por unidade de tempo. 0 seu recíproco, $1 / \gamma$, pode ser identificado como o tempo de residência no compartimento infeccioso, isto é, o tempo médio em que um indivíduo é infecioso. Para a gripe, o período infecioso é tipicamente de 1 a 3 dias. Se o considerarmos igual a 2 dias, por exemplo, isto significa que a taxa de recuperação é $\gamma=1 / 2$ (por indivíduo e por dia) e portanto num dia metade dos infetados recuperam - passam para o compartimento $\mathbf{R}$.

2.7. Vamos agora calcular $\left[\begin{array}{c}\text { Número de suscetíveis } \\ \text { infetados no intervalo }[t, t+\Delta t]\end{array}\right]$. Para modelar este número, podemos pensar da seguinte forma - é claro que o número de indivíduos infetados aumenta quando suscetíveis contraem a doença por contágio (que envolve algum tipo de contacto entre um suscetível e um infetado).

Suponhamos que cada indivduo contacta, por unidade de tempo, em média com c outros, escolhidos aleatoriamente. $c$ diz-se a taxa de contacto per-capita e por unidade de tempo. Pela hipótese [H2] - população homogeneamente misturada - $c$ é constante (uma hipótese muito questionavel!...).

A doença é transmitida apenas quando uma pessoa infeciosa contacta com um suscetível. Se a população total é constituída por $N$ pessoas, a probabilidade de que alguém encontre um suscetível é igual a $S(t) / N$, e portanto, uma pessoa infeciosa tem (em média) contacto com $c S(t) / N$ susceptíveis, por unidade de tempo. Se $p$ é a probabilidade de que um contacto resulte em contágio e, uma vez que existem $I(t)$ indivíduos infetados no total, isso significa que o número de novas infeções, no intervalo de tempo $[t, t+\Delta t]$, é $(c p S(t) I(t) / N) \Delta t$ e podemos escrever

$$
\left[\begin{array}{c}
\text { Número de suscetíveis } \\
\text { infetados no intervalo }[t, t+\Delta t]
\end{array}\right\}=\beta \frac{S(t) I(t)}{N} \Delta t
$$

onde pusemos $\beta=c p$. A constante $\beta$ é chamada o coeficiente de transmissão e tem unidades tempo-1.

Reunindo todas estas informações, obtemos as equações seguintes para as variações do número de suscetíveis, infetados e recuperados no intervalo $[t, t+\Delta t]$ 


$$
\begin{aligned}
\Delta S(t) & =\beta \frac{S(t) I(t)}{N} \Delta t \\
\Delta I(t) & =\left[\beta \frac{S(t) I(t)}{N}-\gamma I(t)\right] \Delta t \\
\Delta R(t) & =-\gamma I(t) \Delta t
\end{aligned}
$$

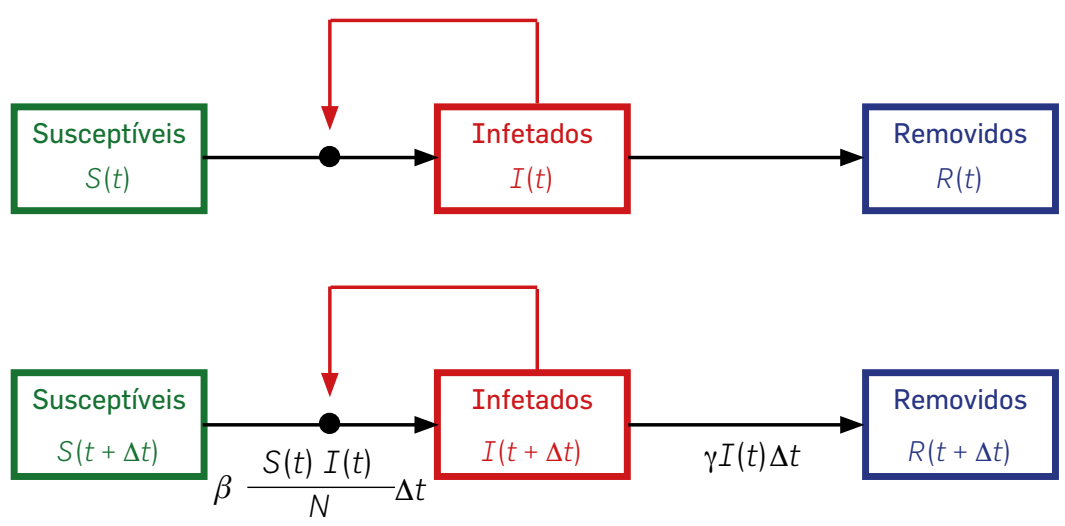

FIGURA 1. Diagrama de fluxo do modelo SIR.

Esta discussão pode ser resumida e visualidada no diagrama de fluxo da Figura 1, cuja explicação deixamos à imaginação do leitor.

Um bom exercício é pensar como mudar as hipóteses simplicadoras noutras mais realistas e desenhar diagramas análogos ao da Figura 1 e, a partir deles, escrever equações análogas às equações SIR (2.6). Um exemplo possível é o da Figura 2 - qual a interpretação?

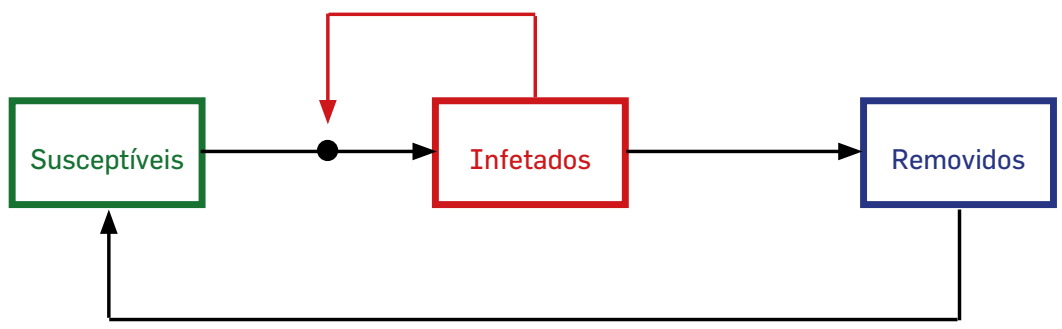

FIGURA 2. Diagrama de fluxo do modelo SIR

\section{UM EXEMPLO CONCRETO}

3.1. Na tabela seguinte mostra-se os números de infectados relativos a um surto de gripe numa escola com $N=763$ alunos, durante 14 dias. 0 gráfico correspondente mostra-se na Figura 3

\begin{tabular}{rcccccccccccccc} 
Dia & 1 & 2 & 3 & 4 & 5 & 6 & 7 & 8 & 9 & 10 & 11 & 12 & 13 & 14 \\
\hline Infetados & 3 & 7 & 22 & 78 & 233 & 300 & 256 & 233 & 189 & 128 & 72 & 33 & 11 & 6
\end{tabular}

Na Figura 3 representam-se os gráficos de $S(n), I(n)$ e $R(n)$, obtidos através das equações 
SIR (2.6), com os valores seguintes para os parâmetros: $\gamma=0.44$ e $\beta=1.68$, correspondentes uma taxa de contacto $c=2$ e a uma probabilidade de contágio $p=0.84$. Como condições iniciais supomos que o surto começou com um único infeccioso em $t_{0}=0$, de tal forma que $I(0)=1$ e $S(0)=762$. Examine detalhadamente o ficheiro Excel ModeloSIR gripe.xlsx. Mude os parâmetros com os cursores lá incluídos, e observe como mudam os gráficos.

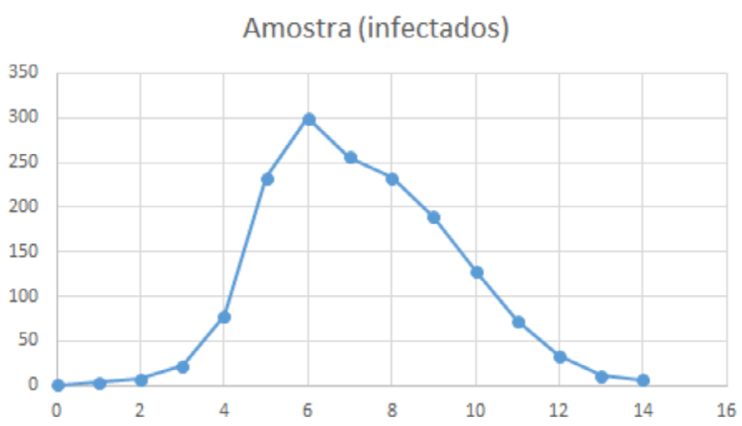

FIGURA 3. Números de infetados relativos a um surto de gripe numa escola com N=763 alunos, durante 14 dias.

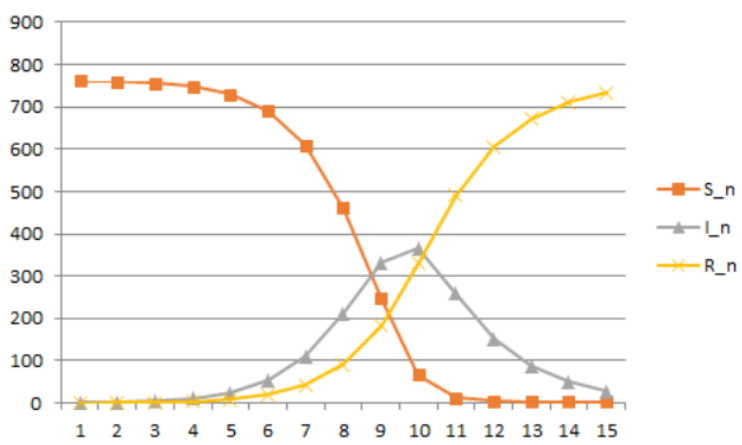

FIGURA 4. Modelo SIR para propagação de gripe. Solução numérica das equações para um surto de gripe num colégio com $N=763$ alunos. 0 surto foi iniciado por um infeccioso, $I(0)=1$, e o número total de suscetíveis $S(0)$ $=762$. Os valores do coeficiente de transmissão e da taxa de remoção foram $\gamma=0.44$ e $\beta=1.68$, respectivamente, correspondentes a uma taxa de contacto $c=2$ e a uma probabilidade de contágio $p=0.84$.

Finalmente na Figura 5 comparam-se os dois gráficos - o de infectados, dados pela amostra, e o de infectados dados pelo modelo das equações SIR. Claro que o ajuste não é perfeito, mas é razoável!... As previsões do modelo parecem concordar bem com os dados da amostra. 0 número de infecciosos começa pequeno, aumenta substancialmente ao longo de 6 dias, e depois diminui gradualmente. Assim, nas últimas fases do surto, há uma possibilidade muito menor de um qualquer infeccioso entrar em contacto com alguém que ainda não foi infectado.

Resumindo: o que temos à partida é

uma amostra, dada pela tabela acima referida, recolhida por observações directas realizadas ao longo de 14 dias, e

- um modelo - o das equações SIR, que deduzimos sob certas hipótese.

Talvez mudando estas hipóteses, que não são de forma alguma inquestionáveis, e portanto o modelo, obtivessemos um mais próximo dos dados reais da amostra. 


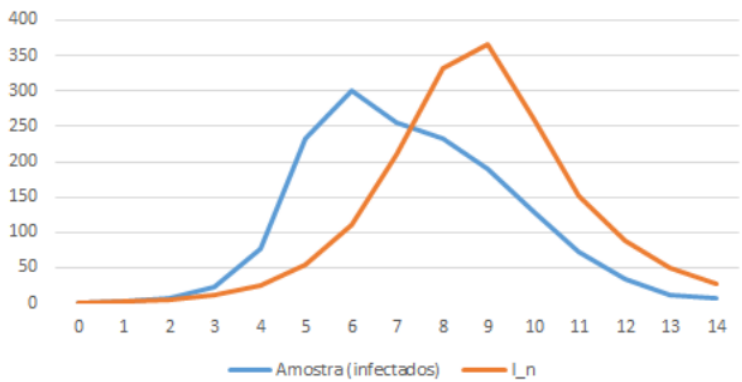

FIGURA 5. Gráficos dos infetados, dados pela amostra, e de infetados dados pelo modelo das equações SIR.

Mas podemos talvez melhorar a situação, escolhendo de forma apropriada os parâmetros que temos ao dispôr - o coeficiente de transmissão $\beta$ e a taxa de recuperação $\gamma$. Existe um método de estimação destes parâmetros, muito usado nestas situações - o chamado método dos mínimos quadrados.

A ideia é escolher os parâmetros $\beta$ e $\gamma$ de tal forma que a função soma dos desvios quadráticos

$$
\mathrm{F}(\beta, \gamma)=\sum_{n=0}^{14}\left[I_{\text {amostra }}(n)-I_{\text {modelo }}(n)\right]^{2}
$$

tenha o menor valor possível.

Veja a Figura 6 e o ficheiro Modelo SIR gripe EMQ.xlsx.

\section{TAXA DE CONTACTO E NÚMERO DE CONTACTO}

4.1. Os modelos de doenças infecciosas são frequentemente formulados de uma maneira um pouco diferente da que fizemos acima - em vez de números absolutos, como antes, usamos agora proporções da população. Para grandes populações, em particular, esta é uma abordagem natural, porque o tamanho exato da população pode não ser conhecido.

4.2. Retomando o modelo SIR, vamos definir

$$
s=\frac{S}{N}, \quad i=\frac{I}{N}, \quad r=\frac{R}{N}
$$

e reconstruir o modelo usando proporções. Observe agora que

$$
s+i+r=1
$$

porque estamos a medir frações da população. Vamos continuar a presumir que não há nascimentos ou imigração, de modo que o tamanho total da população $N$ se mantem constante, apesar de $N$ não aparecer nas nossas equações. Neste contexto, dividindo ambos os membros das equações (2.6) por $N$, obtemos as chamadas equações sir seguintes

$$
\begin{aligned}
& \Delta s(t)=-\beta s(t) i(t) \Delta t \\
& \Delta i(t)=[\beta s(t)-\gamma] i(t) \Delta t \\
& \Delta r(t)=\gamma i(t) \Delta t
\end{aligned}
$$


onde $\beta$ é, como antes, o coeficiente de transmissão.

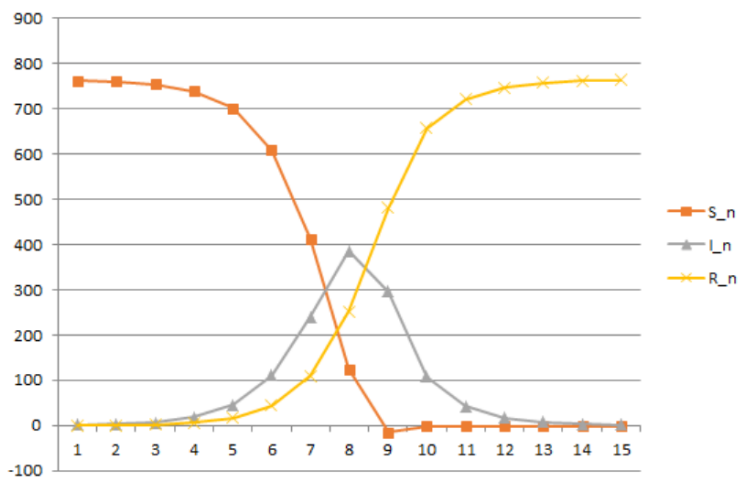

FIGURA 6. Solução numérica das equações para um surto de gripe num colégio com $N=763$ alunos. 0 surto foi iniciado por um infeccioso, $I(0)=1$, e o número total de suscetíveis $S(0)=762$. Os valores do coeficiente de transmissão e da taxa de remoção foram estimados pelo método dos mínimos quadrados como $\beta=2.216 \mathrm{dia}^{-1} \mathrm{e}$ $\gamma=0.59 \mathrm{dia}^{-1}$, respectivamente. Ver ficheiro Excel ModeloSIR gripe EMQ.xlsx.

0 valor de $\beta$ depende é claro das particularidades da doença sob estudo. Por exemplo, como a varicela é altamente contagiosa, parece plausível que uma criança da escola primária possa ter contato, suficiente para espalhar varicela, com quatro outras crianças durante um dia. Neste caso, podemos tomar $\beta=4$ e $\Delta t=1$ dia. Para uma doença menos contagiosa, $\beta$ pode ser um número tão pequeno como 0.02 .

\section{VALORES LIMIAR (THRESHOLD) E PARÂMETROS CRÍTICOS}

5.1. Para analisar o modelo sir e ganhar alguma intuição biológica sobre os parâmetros do modelo, vamos usar as equações (4.2):

$$
\begin{aligned}
& \Delta s(t)=-\beta s(t) i(t) \Delta t \\
& \Delta i(t)=[\beta s(t)-\gamma] i(t) \Delta t \\
& \Delta r(t)=\gamma i(t) \Delta t
\end{aligned}
$$

Diremos que uma epidemia ocorre se $\Delta i(t)>0$, para algum intervalo temporal $[t, t+\Delta t]$ (isto é, se nesse período a proporção de infecciosos cresce). Se $\Delta i(t) \leq 0$ para todos os períodos, o tamanho da classe infecciosa não aumenta e não existe qualquer foco da doença.

5.2. O primeiro passo para a compreensão da dinâmica da doença, é pois entender o sinal de $\Delta i(t)$. Vejamos então como determinar se

$$
\Delta i(t)=[\beta s(t)-\gamma] i(t) \Delta t
$$

é positivo, negativo ou nulo.

É claro que se $i(t)=0$ então $\Delta i(t)=0$ - se não há infectados, não há doença. Suponhamos agora que $i(t)>0$. Então $\Delta i(t)$ será positivo, negativo ou nulo, se o mesmo acontece com $\beta s(t)-\gamma$. Como $\beta>0$, podemos concluir que 


$$
\begin{aligned}
& \text { se } s(t)>\gamma / \beta, \text { então } \Delta i(t)>0 \\
& \text { se } s(t)=\gamma / \beta, \text { então } \Delta i(t)=0 \\
& \text { se } s(t)<\gamma / \beta, \text { então } \Delta i(t)<0
\end{aligned}
$$

5.3. Observe que, a partir de nossas fórmulas originais, temos sempre que $\Delta s(t) \leq 0, \forall t$ e, por isso, a proporção de susceptíveis nunca pode aumentar.

Isto significa que, se a proporção inicial de susceptíveis, $s_{0}$, satisfaz

$$
\mathrm{s}_{0}<\frac{\gamma}{\beta}
$$

então $s(t)<\frac{\gamma}{\beta}$ e portanto, pela terceira implicação de (5.3), $\Delta i(t)<0$ para todo $t$, e a doença diminui - não há epidemia.

No entanto, quando a proporção inicial de susceptíveis, $s_{0}$, satisfaz $s(t)>\frac{\gamma}{\beta}$, o número de infecciosos cresce no primeiro período $[0, \Delta t]$, isto é $\Delta i(0)>0$, o que provoca uma epidemia. Por este motivo, a razão $\frac{\gamma}{\beta}$ é um exemplo de um valor limiar ou crítico (threshold); a relação de $s_{0} \operatorname{com} \frac{\gamma}{\beta}$ é um determinante importante da dinâmica da doença. Como $\frac{\gamma}{\beta}$ representa a taxa de remoção $\gamma$ relativa ao coeficiente de transmissão $\beta$, chamamos-lhe a taxa de remoção relativa.

\section{O NÚMERO BÁSICO DE REPRODUÇÃO $R_{0}$}

6.1. Uma abordagem ligeiramente diferente para o comportamento que descrevemos no ponto anterior, envolve reescrever a equação para $\Delta i(t)$ na forma

$$
\Delta i(t)=\gamma\left(\frac{\beta}{\gamma} s(t)-1\right) i(t) \Delta t
$$

Uma análise semelhante do sinal de $\Delta i(t)$, utilizando esta expressão, mostra que a pergunta $\frac{\beta}{\gamma}$ agora é como a quantidade $s_{0}$ se compara com 1 . Os epidemiologistas chamam à expressão

$$
\mathbb{R}_{0}=\frac{\beta}{\gamma} s_{0}
$$

o número de reprodução básico da infecção.

Da discussão anterior concluímos que se $\mathcal{R}_{0}>1$, então $\Delta i(0)>0$ e portanto ocorre uma epidemia.

$$
\mathbb{R}_{0}=\frac{\beta}{\gamma} s_{0}=\left(\beta s_{0}\right) \frac{1}{\gamma}
$$

de um ponto de vista mais biológico, a fim de compreender o seu nome e importância conceptual. 
Como vimos em (2.5), no modelo SIR o termo

$$
\beta \frac{S(0) I(0)}{N} \Delta t
$$

mede o número de indivíduos infectados, no período inicial $[0, \Delta t]$.

Em particular, pondo $I(0)=1$ e $s_{0}=S(0) / N$, vemos que $\beta s_{0} \Delta t$ é o número de susceptíveis infectados por contacto com um único infeccioso, no período inicial da epidemia. Daí que $\beta s_{0}$ é o número de susceptíveis infetados, por contacto com um único infeccioso, por unidade de tempo, no período inicial da epidemia.

$\mathrm{Na}$ verdade, se introduzirmos um infeccioso numa população de indivíduos todos susceptíveis $(S(0)=N-1, I(0)=1, R(0)=0)$, esse doente infeccioso pode, eventualmente, infectar muitos mais do que $\beta \mathrm{s}_{0}$ susceptíveis, uma vez que ele pode permanecer contagioso por muitos períodos de tempo. Por exemplo, suponhamos que uma criança permanece contagiosa com varicela durante 7 dias. Se usamos 1 dia como período (unidade tempo), a criança poderia infectar $\beta \mathrm{s}_{0} \times 7$ susceptíveis ao longo de uma semana.

Por outro lado, se o período de contágio é de 7 dias, então em cada dia esperamos que aproximadamente $1 / 7$ ou cerca de $14 \%$ do número total de infecciosos se mova da classe I para a classe R. Como a taxa de remoção $\gamma$ mede a fracção da classe infecciosa "curada" durante um único período (1 dia, neste caso), vemos que uma boa estimativa para $\gamma$ é $\gamma \approx 1 / 7 \approx 0.1429$.

Simultanemente, encontramos uma boa interpretação para $1 / \gamma$ - é o tempo médio em que um indivíduo está infeccioso. Na prática, podemos estimar $1 / \gamma$ em doenças reais, observando indivíduos infectados e determinando o tempo médio em que eles permanecem infecciosos.

Concluindo

$$
\mathbb{R}_{0}=\left(\beta S_{0}\right) \frac{1}{\gamma}=\left(\begin{array}{l}
\text { número de novos casos que surgem pela } \\
\text { presença de um infetado, numa população } \\
\text { completamente suscetível, por unidade de } \\
\text { tempo }
\end{array}\right) \times\left(\begin{array}{l}
\text { duração média } \\
\text { da infeção }
\end{array}\right)
$$

6.3. Portanto $\mathcal{R}_{0}$ é interpretado como o número médio de infecções secundárias que seria produzido por um infeccioso numa população total de susceptíveis de tamanho $S(0)$. Note que o valor limiar $\mathcal{R}_{0}=1$ faz todo o sentido do ponto de vista biológico. Se $\mathcal{R}_{0}>1$, então um caso primário de doença gera mais do que um caso secundário da doença, o tamanho da classe infecciosa aumenta, e o resultado é uma epidemia. Se $\mathcal{R}_{0}=1$, então um indivíduo doente produz apenas um novo caso da doença, e nenhuma epidemia pode ocorrer; não pode haver crescimento do número de infecciosos. Quando $R_{0}<1$, a doença morre. Em suma, um epidemia ocorre se e somente se o número de reprodução básica $\boldsymbol{R}_{0}>1$.

6.4. Como o número básico de reprodução tem uma interpretação tão significativa, os epidemiologistas tentar achar uma expressão para $\mathcal{R}_{0}$ para qualquer modelo que eles propõem. Eis algumas estimativas típicas de $\mathcal{R}_{0}$ para algumas doenças infecciosas comuns (na era de pré-vacinação) 


\begin{tabular}{ccc} 
Doença & & $\boldsymbol{R}_{0}$ \\
\hline gripe & $\approx$ & $3-4$ \\
sarampo & $\approx$ & $16-18$ \\
varíola & $\approx$ & 4 \\
varicela & $\approx$ & $10-12$
\end{tabular}

Veja o cálculo de $\mathcal{R}_{0}$ nas folhas Excel ModeloSIR gripe.xlsx e ModeloSIR gripe EMQ.xlsx. Embora num modelo complicado, tal como doenças sexualmente transmissíveis (HIV), podem incluir vários parâmetros adicionais, uma combinação deles deve ser interpretável como um $\mathcal{Z}_{0}$ dessa doença. 0 número de reprodução básico $\mathcal{R}_{0}$ desempenha um papel crucial em decisões de saúde pública, porque um programa de prevenção da doença só será eficaz na prevenção de novos surtos quando ele garante que $R_{0} \leq 1$.

\section{Exemplo}

Suponhamos, para uma determinada doença, um doente infeccioso é introduzido numa população de 500 indivíduos susceptíveis. Vamos usar o modelo SIR, com período de tempo $\Delta t=1$ dia (supondo que é adequado para descrever esta doença). Suponhamos, além disso, que os da- dos indicam que o coeficiente de transmissão é $\beta=0.5$ e que o tempo médio de infecção é de 10 dias, após os quais o doente recupera (ou morre!). Portanto, neste caso, $\gamma=0.1 \mathrm{e}$

$$
R_{0}=\frac{\beta}{\gamma} s_{0} \approx \frac{0.5}{0.1} 1=5
$$

Como $R_{0}=5>1$ espera-se uma epidemia. A folha Excel mostra que o número de infectados tem um pico para $n=21$ ou 22 dias, aproximadamente igual a 250. Metade da população está doente o que traduz uma epidemia severa, sem dúvida.

\section{ESTRATÉGIAS DE IMUNIZAÇÃO. VACINAÇÃO}

7.1. Uma população pode ser protegida da doença de várias maneiras. Por exemplo,

- o número de indivíduos susceptíveis podem ser reduzido através de vacinas,

- a taxa de contacto pode ser reduzida através de quarentenas ou campanhas de saúde pública, ou

- a taxa de remoção pode ser aumentada através de um melhor tratamento médico do doente.

Em suma, uma sociedade pode tentar mudar qualquer um dos parâmetros ou condições iniciais do modelo que descreve a doença.

7.2 Vacinações, quando disponíveis, são uma maneira atraente para controlar a dinâmica da doença, mas os riscos privados e públicos de imunização deve ser equilibrado. Um dos principais objetivos de qualquer programa de imunização é conseguir imunidade global, (ou seja, garantir que nenhuma epidemia possa ocorrer, mesmo se alguns casos da doença estão presentes). No entanto, existem indivíduos que recebem vacinas mas têm algum 
pequeno risco de efeitos secundários.

7.3. Como uma aplicação do número básico de reprodução $\mathcal{R}_{0}$, vamos considerar a vacinação de uma população. Admitindo que seria possível vacinar instantaneamente uma proporção $P$ de uma população, que proporção $P$ devemos vacinar para conseguir a erradicação da doença infecciosa?

A resposta é fácil utilizando o conceito de Ro. Como $\mathcal{R}_{0}=\frac{\beta}{\gamma} S_{0}$, se vacinarmos uma proporção $P$ da população de susceptíveis, então isto implica que o número básico de reprodução muda para

$$
\boldsymbol{R}_{0}=\frac{\beta}{\gamma}(1-P) \mathrm{s}_{0}=(1-P) \boldsymbol{R}_{0}
$$

uma vez que a nova fracção de susceptíves é agora $(1-P) s_{0}$.

Como devemos ter $R_{0}^{\mathrm{vac}}<1$ para erradicação, resolvendo em ordem a $P$, obtemos a fórmula simples

$$
P>1-\frac{1}{R_{0}}
$$

Para a varíola, por exemplo, em que $R_{0} \approx 4$, calculamos $P \approx 75 \%$ da população, mas para o sarampo, em comunidades não vacinadas, onde normalmente $R_{0} \approx 15$, então $P \approx 93 \%$. Está claro deste cálculo porque é que, em todo o mundo, a varíola foi escolhida como alvo prioritário para a erradicação.

7.4. Estudo de imunização em doenças realistas requer o uso de modelos mais complicados. A dinâmica da doença, muitas vezes é diferente conforme a idade, estrato social, e outros factores, e, por isso, é usual dividir a população de susceptíveis, infecciosos e removidos em vários estratos ou subgrupos.

Um modelo pode dividir a população em vários subgrupos por idade, sexo ou outros fatores, e ser usado para determinar quais grupos devem ser alvo de uma campanha de imunização. Considerações sociais e médicas são cruciais.

\section{REFERÊNCIAS}

${ }^{1}$ https://pt.wikipedia.org/wiki/Peste_negra 